\title{
On the two-parameter Lorentzian homothetic motions
}

\author{
Muhsin Çelik*, Doğan Ünal and Mehmet Ali Güngör
}

\section{"Correspondence:}

mcelik@sakarya.edu.tr

Department of Mathematics,

Faculty of Arts and Sciences,

Sakarya University, Sakarya, Turkey

\begin{abstract}
In this study, sliding velocity, pole lines, hodograph, and acceleration poles of two-parameter Lorentzian homothetic motions at $\forall(\lambda, \mu)$ positions are obtained. By defining two-parameter Lorentzian homothetic motion along a curve in Lorentzian space $L^{3}$, the theorems related to this motion and characterizations of the trajectory surface are given.
\end{abstract}

MSC: 53A17;53B30; $14 \mathrm{H} 50$

Keywords: two-parameter motion; planar motion; Lorentz plane and space

\section{Introduction}

To investigate the geometry of the motion of a line or a point in the motion of space is important in the study of space kinematics or spatial mechanisms or in physics. The geometry of such a motion of a point or line has a number of applications in geometric modeling and model-bored manufacturing of mechanical products or in the design of robotic motion. These are specifically used to generate geometric models of shell-type objects and thick surfaces [1-3].

Muller has introduced one- and two-parameter planar motions and obtained the relations between absolute, relative, sliding velocity, and pole curves of these motions [4]. Moreover, two-parameter motions in three-dimensional space are defined by [5] and [6]. Lorentzian metric in three-dimensional Minkowski space $L^{3}$ is indefinite. In the theory of relativity, the geometry of indefinite metric is very crucial. Thus, by taking a Lorentzian plane $L^{2}$ instead of an Euclidean plane $E^{2}$, Ergin [7] has introduced one-parameter planar motion in the Lorentzian plane. In [8] all one-parameter motions obtained from twoparameter motion on the Lorentzian plane are investigated.

In this paper, firstly we introduce two-parameter homothetic motions in a Lorentzian plane $L^{2}$ and we calculate the pole points obtained from Lorentzian homothetic motion. Additionally, we give some theorems and corollaries as regards these pole points. Similarly, we calculate the acceleration poles of Lorentzian homothetic motions. By considering the above mentioned, we define two-parameter homothetic motion along a curve in Lorentzian space $L^{3}$ and we give the equation of the trajectory surfaces formed by Lorentzian homothetic motions. Finally, we obtain the parametrizations of the trajectory surfaces and give some examples for these trajectory surfaces.

○2014 Celik et al.; licensee Springer. This is an Open Access article distributed under the terms of the Creative Commons Attribution License (http://creativecommons.org/licenses/by/2.0), which permits unrestricted use, distribution, and reproduction in any medium, provided the original work is properly cited. 


\section{Two-parameter homothetic motions in Lorentzian plane}

The Lorentzian homothetic motion is examined by

$$
Y=h A X+C
$$

for $h(\lambda, \mu) \neq$ const. Also, there can be given some special results of $(\lambda, \mu)=(0,0)$ and $h(\lambda, \mu)$.

Definition 2.1 In a Lorentzian plane, general two-parameter homothetic motion is defined by

$$
\left[\begin{array}{l}
y_{1} \\
y_{2}
\end{array}\right]=h(\lambda, \mu)\left[\begin{array}{ll}
\operatorname{ch} \theta(\lambda, \mu) & \operatorname{sh} \theta(\lambda, \mu) \\
\operatorname{sh} \theta(\lambda, \mu) & \operatorname{ch} \theta(\lambda, \mu)
\end{array}\right]\left[\begin{array}{l}
x \\
y
\end{array}\right]+\left[\begin{array}{l}
a(\lambda, \mu) \\
b(\lambda, \mu)
\end{array}\right],
$$

where $\left(y_{1}, y_{2}\right)$ and $(x, y)$ are coordinate functions of the fixed $L^{\prime 2}$ plane and moving $L^{2}$ planes, respectively. If $\lambda$ and $\mu$ in $C^{\infty}$ are given by the differential functions of the time parameter $t$, then homothetic motions $M_{I}$ are obtained and called homothetic motions $M_{I}$ obtained from homothetic motions $M_{I I}$ on the Lorentzian plane.

Here, at the initial time $(\lambda, \mu)=(0,0)$ and $\theta(0,0)=a(0,0)=b(0,0)=(0,0)$, the coordinate systems of the moving $L^{2}$ and fixed $L^{\prime 2}$ planes are congruent.

Theorem 2.1 The equation of the pole points of Lorentzian homothetic motions $M_{I}$ obtained from Lorentzian homothetic motions $M_{I I}$ on a moving plane is

$$
\begin{aligned}
& (h \dot{a} \dot{\theta} \operatorname{ch} \theta+\dot{h} \dot{a} \operatorname{sh} \theta-h \dot{b} \dot{\theta} \operatorname{sh} \theta-\dot{h} \dot{b} \operatorname{ch} \theta) x_{p} \\
& \quad+(h \dot{a} \dot{\theta} \operatorname{sh} \theta+\dot{h} \dot{a} \operatorname{ch} \theta-h \dot{b} \dot{\theta} \operatorname{ch} \theta-\dot{h} \dot{b} \operatorname{sh} \theta) y_{p}=0,
\end{aligned}
$$

when $|\dot{h}| \neq|h \dot{\theta}|$.

Proof By differentiating equation (2.2) with respect to $t$ and simplifying it, we obtain

$$
\begin{aligned}
& x_{p}=\frac{-h \dot{a} \dot{\theta} \operatorname{sh} \theta-\dot{h} \dot{a} \operatorname{ch} \theta+h \dot{b} \dot{\theta} \operatorname{ch} \theta+\dot{h} \dot{b} \operatorname{sh} \theta}{\dot{h}^{2}-h^{2} \dot{\theta}^{2}}, \\
& y_{p}=\frac{h \dot{a} \dot{\theta} \operatorname{ch} \theta+\dot{h} \dot{a} \operatorname{sh} \theta-h \dot{b} \dot{\theta} \operatorname{sh} \theta-\dot{h} \dot{b} \operatorname{ch} \theta}{\dot{h}^{2}-h^{2} \dot{\theta}^{2}} .
\end{aligned}
$$

After some routine calculations, the equation of the pole points (2.3) is obtained. The pole points of Lorentzian homothetic motions $M_{I}$ obtained from Lorentzian homothetic motions $M_{I I}$ on a moving plane are given by

$$
P\left(x_{p}, y_{p}\right)=\left(\frac{h \dot{b} \dot{\theta}-\dot{h} \dot{a}}{\dot{h}^{2}-h^{2} \dot{\theta}^{2}}, \frac{h \dot{a} \dot{\theta}-\dot{h} \dot{b}}{\dot{h}^{2}-h^{2} \dot{\theta}^{2}}\right)
$$

at the position of $(\lambda, \mu)=(0,0)$ and the equation of the pole points is

$$
(h \dot{a} \dot{\theta}-\dot{h} \dot{b}) x_{p}-(h \dot{b} \dot{\theta}-\dot{h} \dot{a}) y_{p}=0 .
$$


The pole points of Lorentzian homothetic motions $M_{I}$ obtained from Lorentzian homothetic motions $M_{I I}$ on a moving plane at the position of $(\lambda, \mu)=(0,0)$ give the following results.

Corollary 2.1 If $\theta(\lambda, \mu)=$ const, then the pole points lie on the line

$$
\left(h_{\mu} b_{\lambda}-h_{\lambda} b_{\mu}\right) x_{p}+\left(h_{\lambda} a_{\mu}-h_{\mu} a_{\lambda}\right) y_{p}=a_{\lambda} b_{\mu}-a_{\mu} b_{\lambda} .
$$

Corollary 2.2 If $h(\lambda, \mu) \neq 0$ is a constant, then the pole points lie on the line

$$
\left(a_{\mu} \theta_{\lambda}-a_{\lambda} \theta_{\mu}\right) x_{p}-\left(b_{\mu} \theta_{\lambda}-b_{\lambda} \theta_{\mu}\right) y_{p}=\frac{1}{h}\left(a_{\lambda} b_{\mu}-a_{\mu} b_{\lambda}\right) .
$$

Corollary 2.3 If $h(\lambda, \mu)=1$, then the pole points lie on the line

$$
\left(a_{\mu} \theta_{\lambda}-a_{\lambda} \theta_{\mu}\right) x_{p}-\left(b_{\mu} \theta_{\lambda}-b_{\lambda} \theta_{\mu}\right) y_{p}=a_{\lambda} b_{\mu}-a_{\mu} b_{\lambda}, \quad \text { [8]. }
$$

Theorem 2.2 The equation of the pole points of Lorentzian homothetic motions $M_{I}$ obtained from Lorentzian homothetic motions $M_{I I}$ on a fixed plane is

$$
(h \dot{a} \dot{\theta}-\dot{h} \dot{b}) \bar{x}_{p}-(h \dot{b} \dot{\theta}-\dot{h} \dot{a}) \bar{y}_{p}=a(h \dot{a} \dot{\theta}-\dot{h} \dot{b})-b(h \dot{b} \dot{\theta}-\dot{h} \dot{a}),
$$

at the position of $\lambda=\mu=0$ and when $|\dot{h}| \neq|h \dot{\theta}|$.

Proof By taking $P\left(x_{p}, y_{p}\right)$ in equation (2.1), we have the pole points

$$
\bar{P}\left(\bar{x}_{p}, \bar{y}_{p}\right)=\left(\frac{h^{2} \dot{b} \dot{\theta}-\dot{h} h \dot{a}}{\dot{h}^{2}-h^{2} \dot{\theta}^{2}}+a, \frac{h^{2} \dot{a} \dot{\theta}-\dot{h} h \dot{b}}{\dot{h}^{2}-h^{2} \dot{\theta}^{2}}+b\right)
$$

and the equation of the pole points (2.10) is obtained. The pole points of Lorentzian homothetic motions $M_{I}$ obtained from Lorentzian homothetic motions $M_{I I}$ on a fixed plane at the position of $(\lambda, \mu)=(0,0)$ give the following results.

Corollary 2.4 On the fixed plane $\theta(\lambda, \mu)=$ const, the pole points lie on the line

$$
\left(h_{\mu} b_{\lambda}-h_{\lambda} b_{\mu}\right) \bar{x}_{p}+\left(h_{\lambda} a_{\mu}-h_{\mu} a_{\lambda}\right) \bar{y}_{p}=h\left(a_{\lambda} b_{\mu}-a_{\mu} b_{\lambda}\right) .
$$

Corollary 2.5 As a special case in Corollary 2.4, if $h(\lambda, \mu)=1$, the pole points of the fixed and moving planes are congruent.

Corollary 2.6 If $h(\lambda, \mu) \neq 0$ is constant, the pole points of fixed planes lie on the line equation (2.9) [8].

Corollary 2.7 As a special case in Corollary 2.2, if $h(\lambda, \mu)=1$, the pole points of moving planes are congurent to pole lines of fixed plane in Corollary 2.6.

If the pole points of Lorentzian homothetic motions $M_{I}$ obtained from Lorentzian homothetic motions $M_{I I}$ are chosen y axis, then $x_{p}=0$ at the position of $\lambda=\mu=0$. Hence, 
we have

$$
y_{p}=-\frac{\dot{a}}{h \dot{\theta}} .
$$

Therefore, there is a relation between the pole lines of the fixed plane and the pole lines of a moving plane as follows:

$$
\bar{y}_{p}=h y_{p} .
$$

Now, we investigate the sliding velocity $\vec{V}_{f}=\left(\dot{y}_{1}, \dot{y}_{2}\right)$ of any points $B(x, y)$ at the position of $\lambda=\mu=0$. Equation (2.2) is derived with respect to $t$ and with the position of $\lambda=\mu=0$, we have

$$
\begin{aligned}
& \dot{y}_{1}=\dot{h} x+h \dot{\theta} y+\dot{a}, \\
& \dot{y}_{2}=\dot{h} y+h \dot{\theta} x+\dot{b} .
\end{aligned}
$$

Thus, the sliding velocity is obtained as follows:

$$
\vec{V}_{f}=(\dot{h} x+h \dot{\theta} y+\dot{a}, \dot{h} y+h \dot{\theta} x+\dot{b}) .
$$

Theorem 2.3 In Lorentzian homothetic motions $M_{I}$ obtained from Lorentzian homothetic motions $M_{I I}$, let $y$-axis be the pole axis at the position of $\lambda=\mu=0$. Then, the relation between the pole ray going from the pole point $P\left(x_{p}, y_{p}\right)$ to the point $B(x, y)$ and the sliding velocity $\vec{V}_{f}$ of the point $B(x, y)$ is

$$
\left\langle\vec{V}_{f}, \overrightarrow{P B}\right\rangle_{L}=\dot{h}\left(x^{2}-y^{2}\right)-2 \dot{b} y-\frac{\dot{a} \dot{b}}{h \dot{\theta}} .
$$

Proof By reason of the fact that the pole axis is an $y$-axis, we have $\left(x_{p}, y_{p}\right)=\left(0,-\frac{\dot{a}}{h \dot{\theta}}\right)$ and $\overrightarrow{P B}=\left(x, y+\frac{\dot{a}}{h \theta}\right)$ from equation (2.5). Then it is seen that

$$
\begin{aligned}
\left\langle\vec{V}_{f}, \overrightarrow{P B}\right\rangle_{L} & =\left\langle(\dot{h} x+h \dot{\theta} y+\dot{a}, \dot{h} y+h \dot{\theta} x+\dot{b}),\left(x, y+\frac{\dot{a}}{h \dot{\theta}}\right)\right\rangle_{L} \\
& =\dot{h}\left(x^{2}-y^{2}\right)-2 \dot{b} y-\frac{\dot{a} \dot{b}}{h \dot{\theta}} .
\end{aligned}
$$

Corollary 2.8 If $h(\lambda, \mu)$ is a constant never vanishing and the pole axis is the $y$-axis, then the pole ray and the sliding velocity $\vec{V}_{f}$ are perpendicular [8].

Theorem 2.4 The length of the sliding velocity vector of Lorentzian homothetic motions $M_{I}$ obtained from Lorentzian homothetic motion $M_{I I}$ is

$$
\left\|\vec{V}_{f}\right\|_{L}=\sqrt{\left|\dot{h}^{2}-h^{2} \dot{\theta}^{2}\right|}\|\overrightarrow{P B}\|_{L}
$$

at the position of each $(\lambda, \mu)$. 
Proof Substituting the differentiation of $C$ given in equation (2.1) into $\vec{V}_{f}$, we get

$$
\vec{V}_{f}=\left(\begin{array}{l}
(\dot{h} \operatorname{ch} \theta+h \dot{\theta} \operatorname{sh} \theta)\left(x-x_{p}\right)+(\dot{h} \operatorname{sh} \theta+h \dot{\theta} \operatorname{ch} \theta)\left(y-y_{p}\right) \\
(\dot{h} \operatorname{sh} \theta+h \dot{\theta} \operatorname{ch} \theta)\left(x-x_{p}\right)+(\dot{h} \operatorname{ch} \theta+h \dot{\theta} \operatorname{sh} \theta)\left(y-y_{p}\right)
\end{array}\right) .
$$

Then, the length of the sliding velocity vector $\vec{V}_{f}$ is obtained.

Corollary 2.9 If $h(\lambda, \mu)=1$, then we obtain $\left\|\vec{V}_{f}\right\|_{L}=|\dot{\theta}|\|\overrightarrow{P B}\|_{L}[8]$.

Theorem 2.5 For all Lorentzian homothetic motions $M_{I}$ obtained from Lorentzian homothetic motions $M_{I I}$, let $\psi$ be angle between the pole ray going from the pole point $P$ to the point $B$ and the sliding velocity vector $\vec{V}_{f}$. Then we have the relation

$$
\operatorname{ch} \psi(\lambda, \mu)=-\frac{\dot{h} \operatorname{ch} \theta+h \dot{\theta} \operatorname{sh} \theta}{\sqrt{\left|\dot{h}^{2}-h^{2} \dot{\theta}^{2}\right|}}
$$

at the position of each $(\lambda, \mu)$.

Proof There is the following relation between the pole ray $\overrightarrow{P B}=\left(x-x_{p}, y-y_{p}\right)$ and sliding velocity vector $\vec{V}_{f}$ :

$$
\left\langle\overrightarrow{P B}, \vec{V}_{f}\right\rangle_{L}=(\dot{h} \operatorname{ch} \theta+h \dot{\theta} \operatorname{sh} \theta)\|\overrightarrow{P B}\|_{L}^{2}
$$

On the other hand,

$$
\left\langle\overrightarrow{P B}, \vec{V}_{f}\right\rangle_{L}=-\left\|\vec{V}_{f}\right\|_{L}\|\overrightarrow{P B}\|_{L} \operatorname{ch} \psi(\lambda, \mu)
$$

From the equality of the last two equations, we obtain equation (2.17).

Corollary 2.10 If $h(\lambda, \mu) \neq 0$ is constant, then we obtain

$$
\psi(\lambda, \mu)=\frac{\pi}{2}+\theta(\lambda, \mu), \quad \theta=2 k \pi(k=0,1, \ldots) \quad[8] .
$$

Definition 2.2 When the sliding velocity vectors of a fixed point are carried to the initial point, without changing the directions, then the locus of the end points of these vectors is a curve called a hodograph.

Now we investigate any $(x, y)$ points of the locus of the hodographs in all Lorentzian homothetic motions $M_{I}$ obtained from Lorentzian homothetic motion $M_{I I}$, according to the position of $\dot{\lambda}$ and $\dot{\mu}$. For this let $\dot{\mu}^{2}-\dot{\lambda}^{2}=1$. By taking the derivatives with respect to $t$ of equation (2.2), we have

$$
\begin{aligned}
\dot{y}_{1}= & \left(h_{\lambda} x \operatorname{ch} \theta+h_{\lambda} y \operatorname{sh} \theta+h \theta_{\lambda} x \operatorname{sh} \theta+h \theta_{\lambda} y \operatorname{ch} \theta+a_{\lambda}\right) \dot{\lambda} \\
& +\left(h_{\mu} x \operatorname{ch} \theta+h_{\mu} y \operatorname{sh} \theta+h \theta_{\mu} x \operatorname{sh} \theta+h \theta_{\mu} y \operatorname{ch} \theta+a_{\mu}\right) \dot{\mu}, \\
\dot{y}_{2}= & \left(h_{\lambda} x \operatorname{sh} \theta+h_{\lambda} y \operatorname{ch} \theta+h \theta_{\lambda} x \operatorname{ch} \theta+h \theta_{\lambda} y \operatorname{sh} \theta+b_{\lambda}\right) \dot{\lambda} \\
& +\left(h_{\mu} x \operatorname{sh} \theta+h_{\mu} y \operatorname{ch} \theta+h \theta_{\mu} x \operatorname{ch} \theta+h \theta_{\mu} y \operatorname{sh} \theta+b_{\mu}\right) \dot{\mu} .
\end{aligned}
$$


Let us investigate the solution of the last equation system by taking $(\lambda, \mu)=(0,0)$ for simplicity. From equation (2.18), we find

$$
\begin{aligned}
\operatorname{det} \Delta= & h\left(x^{2}-y^{2}\right)\left(h_{\lambda} \theta_{\mu}-h_{\mu} \theta_{\lambda}\right)+h x\left(a_{\lambda} \theta_{\mu}-a_{\mu} \theta_{\lambda}\right)-h y\left(b_{\lambda} \theta_{\mu}-b_{\mu} \theta_{\lambda}\right) \\
& +x\left(h_{\lambda} b_{\mu}-h_{\mu} b_{\lambda}\right)-y\left(h_{\lambda} a_{\mu}-h_{\mu} a_{\lambda}\right)+a_{\lambda} b_{\mu}-a_{\mu} b_{\lambda},
\end{aligned}
$$

that is,

$$
\begin{aligned}
& {\left[h^{2} x^{2}\left(\theta_{\lambda}^{2}-\theta_{\mu}^{2}\right)+y^{2}\left(h_{\lambda}^{2}-h_{\mu}^{2}\right)+2 h x y\left(h_{\lambda} \theta_{\lambda}-h_{\mu} \theta_{\mu}\right)+2 h x\left(b_{\lambda} \theta_{\lambda}-b_{\mu} \theta_{\mu}\right)\right.} \\
& \left.\quad+2 y\left(h_{\lambda} b_{\lambda}-h_{\mu} b_{\mu}\right)+\left(b_{\lambda}^{2}-b_{\mu}^{2}\right)\right] \dot{y}_{1}^{2}+\left[x^{2}\left(h_{\lambda}^{2}-h_{\mu}^{2}\right)+h^{2} y^{2}\left(\theta_{\lambda}^{2}-\theta_{\mu}^{2}\right)\right. \\
& \left.\quad+2 h x y\left(h_{\lambda} \theta_{\lambda}-h_{\mu} \theta_{\mu}\right)+2 x\left(h_{\lambda} a_{\lambda}-h_{\mu} a_{\mu}\right)+2 h y\left(a_{\lambda} \theta_{\lambda}-a_{\mu} \theta_{\mu}\right)+\left(a_{\lambda}^{2}-a_{\mu}^{2}\right)\right] \dot{y}_{2}^{2} \\
& \quad-2\left[h\left(x^{2}+y^{2}\right)\left(h_{\lambda} \theta_{\lambda}-h_{\mu} \theta_{\mu}\right)+x y\left(h_{\lambda}^{2}-h_{\mu}^{2}+h^{2}\left(\theta_{\lambda}^{2}-\theta_{\mu}^{2}\right)\right)+h x\left(a_{\lambda} \theta_{\lambda}-a_{\mu} \theta_{\mu}\right)\right. \\
& \left.\quad+h y\left(b_{\lambda} \theta_{\lambda}-b_{\mu} \theta_{\mu}\right)+x\left(h_{\lambda} b_{\lambda}-h_{\mu} b_{\mu}\right)+y\left(h_{\lambda} a_{\lambda}-h_{\mu} a_{\mu}\right)+a_{\lambda} b_{\lambda}-a_{\mu} b_{\mu}\right] \dot{y}_{1} \dot{y}_{2} \\
& =(\operatorname{det} \Delta)^{2} .
\end{aligned}
$$

Finally, if we find the values of $\dot{\lambda}$ and $\dot{\mu}$ and substitute these values into the equation $\dot{\mu}^{2}-$ $\dot{\lambda}^{2}=1$, and the following theorem is found.

Theorem 2.6 In all Lorentzian homothetic motions $M_{I}$ obtained from Lorentzian homothetic motions $M_{I I}$, the locus of the hodograph is a hyperbola at the position of $\lambda=\mu=0$.

Proof Setting $\lambda=\mu=0$ in equation (2.19) and taking into consideration the general conic form, we can say that

$$
A x^{2}+2 B x y+C y^{2}+2 D x+2 E y+F=0
$$

and

$$
\operatorname{det}\left|\begin{array}{ll}
A & B \\
B & C
\end{array}\right|=\left(\begin{array}{l}
h\left(x^{2}+y^{2}\right)\left(h_{\lambda} \theta_{\lambda}-h_{\mu} \theta_{\mu}\right)+h x\left(a_{\lambda} \theta_{\mu}-a_{\mu} \theta_{\lambda}\right)+h y\left(b_{\lambda} \theta_{\mu}-b_{\mu} \theta_{\lambda}\right) \\
-y\left(h_{\lambda} a_{\mu}-h_{\mu} a_{\lambda}\right)+x\left(h_{\lambda} b_{\mu}-h_{\mu} b_{\lambda}\right)+\left(a_{\lambda} b_{\mu}-a_{\mu} b_{\lambda}\right)
\end{array}\right)^{2}<0 .
$$

That is, the locus of the hodograph is a hyperbola.

\section{The acceleration pole of the homothetic motions}

Now we will investigate the locus of the points which have zero sliding acceleration. So, we need to solve the equation $(\ddot{h} A+h \ddot{A}+2 \dot{h} \dot{A}) X+\ddot{C}=0$. The solution of this equation gives the coordinates of the acceleration pole points. From this we get

$$
\begin{aligned}
x_{i p} & =\frac{\ddot{a}\left(-\ddot{h} \operatorname{ch} \theta-h \dot{\theta}^{2} \operatorname{ch} \theta-h \ddot{\theta} \operatorname{sh} \theta-2 \dot{h} \dot{\theta} \operatorname{sh} \theta\right)+\ddot{b}\left(\ddot{h} \operatorname{sh} \theta+h \dot{\theta}^{2} \operatorname{sh} \theta+h \ddot{\theta} \operatorname{ch} \theta+2 \dot{h} \dot{\theta} \operatorname{ch} \theta\right)}{\left(\ddot{h}+h \dot{\theta}^{2}\right)^{2}-(2 \dot{h} \dot{\theta}+h \ddot{\theta})^{2}}, \\
y_{i p} & =\frac{\ddot{a}\left(\ddot{h} \operatorname{sh} \theta+h \dot{\theta}^{2} \operatorname{sh} \theta+h \ddot{\theta} \operatorname{ch} \theta+2 \dot{h} \dot{\theta} \operatorname{ch} \theta\right)+\ddot{b}\left(-\ddot{h} \operatorname{ch} \theta-h \dot{\theta}^{2} \operatorname{ch} \theta-h \ddot{\theta} \operatorname{sh} \theta-2 \dot{h} \dot{\theta} \operatorname{sh} \theta\right)}{\left(\ddot{h}+h \dot{\theta}^{2}\right)^{2}-(2 \dot{h} \dot{\theta}+h \ddot{\theta})^{2}} .
\end{aligned}
$$

Thus, for $\lambda=\mu=0$, the acceleration pole points are given by

$$
P\left(x_{i p}, y_{i p}\right)=\left(\frac{2 \dot{h} \ddot{b} \dot{\theta}-\ddot{h} \ddot{a}+h\left(\ddot{b} \ddot{\theta}-\ddot{a} \dot{\theta}^{2}\right)}{\left(\ddot{h}+h \dot{\theta}^{2}\right)^{2}-(2 \dot{h} \dot{\theta}+h \ddot{\theta})^{2}}, \frac{2 \dot{h} \ddot{a} \dot{\theta}-\dddot{h} \ddot{b}+h\left(\ddot{a} \ddot{\theta}-\ddot{b} \dot{\theta}^{2}\right)}{\left(\ddot{h}+h \dot{\theta}^{2}\right)^{2}-(2 \dot{h} \dot{\theta}+h \ddot{\theta})^{2}}\right) .
$$


Theorem 3.1 The equation of the acceleration poles of the Lorentzian homothetic motions $M_{I}$ obtained from Lorentzian homothetic motions $M_{I I}$ on the moving plane is

$$
(h \ddot{a} \ddot{\theta}-\dddot{h} \ddot{b}) x_{i p}-(h \ddot{b} \ddot{\theta}-\ddot{h} \ddot{a}) y_{i p}=0
$$

at position $\lambda=\mu=\dot{\lambda}=\dot{\mu}=0$ and when $\left|\ddot{h}+h \dot{\theta}^{2}\right| \neq|2 \dot{h} \dot{\theta}+h \ddot{\theta}|$.

Proof Setting $\lambda=\mu=\dot{\lambda}=\dot{\mu}=0$ in equation (3.1) gives us the desired equation. Therefore, we can give the following corollaries at the position of $(\lambda, \mu)=(0,0)$.

Corollary 3.1 The acceleration pole points on the moving plane lie on the line given by equation (2.7) if $\theta(\lambda, \mu)$ is constant.

Corollary 3.2 The acceleration pole points on the moving plane lie on the line given by equation (2.8) if $h(\lambda, \mu) \neq 0$ is constant.

Corollary 3.3 The acceleration pole points on the moving plane lie on the line given by equation (2.9) if $h(\lambda, \mu)=1$, [8].

Corollary 3.4 If $h(\lambda, \mu) \neq 0$ is constant, the pole line on the moving planes obtained from Corollary 2.2 and the acceleration pole line on the moving planes obtained from Corollary 3.2 are congruent [8].

Theorem 3.2 The equation of the acceleration pole points of the Lorentzian homothetic motions $M_{I}$ obtained from Lorentzian homothetic motions $M_{I I}$ on the fixed plane is

$$
(h \ddot{a} \ddot{\theta}-\dddot{h} \ddot{b}) \bar{x}_{i p}-(h \ddot{b} \ddot{\theta}-\ddot{h} \ddot{a}) \bar{y}_{i p}=0
$$

at position $\lambda=\mu=\dot{\lambda}=\dot{\mu}=0$ and when $\left|\ddot{h}+h \dot{\theta}^{2}\right| \neq|2 \dot{h} \dot{\theta}+h \ddot{\theta}|$.

Proof If we substitute the acceleration pole points into equation (2.1), we find

$$
\bar{P}\left(\bar{x}_{i p}, \bar{y}_{i p}\right)=\left(h \frac{2 \dot{h} \ddot{b} \dot{\theta}-\ddot{h} \ddot{a}+h\left(\ddot{b} \ddot{\theta}-\ddot{a} \dot{\theta}^{2}\right)}{\left(\ddot{h}+h \dot{\theta}^{2}\right)^{2}-(2 \dot{h} \dot{\theta}+h \ddot{\theta})^{2}}+a, h \frac{2 \dot{h} \ddot{a} \dot{\theta}-\dddot{h} \ddot{b}+h\left(\ddot{a} \ddot{\theta}-\ddot{b} \dot{\theta}^{2}\right)}{\left(\ddot{h}+h \dot{\theta}^{2}\right)^{2}-(2 \dot{h} \dot{\theta}+h \ddot{\theta})^{2}}+b\right) .
$$

If we take $\lambda=\mu=\dot{\lambda}=\dot{\mu}=0$ in the last equation, we have equation (3.4).

So, we can give the following corollaries at the position of $\lambda=\mu=\dot{\lambda}=\dot{\mu}=0$.

Corollary 3.5 The acceleration pole points on the fixed plane lie on the line given by equation (2.12) if $\theta(\lambda, \mu)$ is constant.

Corollary 3.6 As a special case, if $h(\lambda, \mu)=1$ and $\theta(\lambda, \mu)$ is constant, the acceleration pole points on the moving plane and the acceleration pole points on the fixed plane are congruent.

Corollary 3.7 The acceleration pole points on the fixed plane lie on the line given by equation (2.9) if $h(\lambda, \mu) \neq 0$ is constant. 
Corollary 3.8 If $h(\lambda, \mu)=1$ the acceleration pole line of a moving plane obtained from Corollary 3.2 and the acceleration pole line of a fixed plane obtained from Corollary 3.5 are congruent.

Corollary 3.9 As is seen from Corollaries 2.4, 3.5 and 2.6, 3.7, the pole line of a fixed plane and the acceleration pole line of a fixed plane are congruent.

\section{Two-parameter homothetic motion along a curve in Lorentzian space}

In this section, we define two-parameter homothetic motion along a curve in a Lorentzian space and obtain the characterization of the same trajectory surface.

Let $L^{3}$ be a Lorentz 3-space endowed with a Lorentzian inner product $\langle,\rangle_{L}$ of signature $(-,+,+)$. A vector $\vec{x}=\left(x_{1}, x_{2}, x_{3}\right)$ of $L^{3}$ is said to be timelike if $\langle\vec{x}, \vec{x}\rangle_{L}<0$, spacelike if $\langle\vec{x}, \vec{x}\rangle_{L}>0$ and lightlike (or null) if $\langle\vec{x}, \vec{x}\rangle_{L}=0$. The set of all vectors $\vec{x}$ such that $\langle\vec{x}, \vec{x}\rangle_{L}=0$ is called the lightlike (or null) cone and is denoted by $\Gamma$. The norm of a vector $\vec{x}$ is defined to be $\|\vec{x}\|=\sqrt{\left|\langle\vec{x}, \vec{x}\rangle_{L}\right|}$. The Lorentz vector product of the vector $\vec{x}$ and $\vec{y}$ is defined by

$$
\vec{x} \wedge_{L} \vec{y}=\left(x_{2} y_{3}-x_{3} y_{2}, x_{1} y_{3}-x_{3} y_{1}, x_{2} y_{1}-x_{1} y_{2}\right) .
$$

This yields

$$
\vec{e}_{1} \wedge_{L} \vec{e}_{2}=-\vec{e}_{3}, \quad \vec{e}_{2} \wedge_{L} \vec{e}_{3}=\vec{e}_{1}, \quad \vec{e}_{3} \wedge_{L} \vec{e}_{1}=-\vec{e}_{2},
$$

where $\vec{e}_{1}, \vec{e}_{2}, \vec{e}_{3}$ are the basis of the space $L^{3}$ [9]. Semi-orthogonal matrices provide a rotation by the angle (hyperbolic) $t$ around the vector $\vec{w}$. The shape of the matrix depends on the type of the vector $\vec{w}$ as seen in [10].

Let $\vec{w}(s)=\left(w_{1}(s), w_{2}(s), w_{3}(s)\right)$ be spacelike (or timelike) vector which is a differentiable function with respect to $s \in \mathbb{R}$, a vector-valued function. Accordingly, there is a unique $\Omega$ semi-skew symmetric matrix

$$
\Omega(1,3)=\left\{\begin{array}{l}
\Omega \in \mathbb{R}_{3}^{3}, \quad \Omega^{T}=-\varepsilon \Omega \varepsilon, \quad \Omega=\left[\begin{array}{ccc}
0 & w_{3} & -w_{2} \\
w_{3} & 0 & -w_{1} \\
-w_{2} & w_{1} & 0
\end{array}\right] \\
w_{i} \in \mathbb{R}, \quad \varepsilon=\left[\begin{array}{ccc}
-1 & 0 & 0 \\
0 & 1 & 0 \\
0 & 0 & 1
\end{array}\right]
\end{array}\right\}
$$

for all $\forall s \in I \subset \mathbb{R}$ satisfying the following equality:

$$
\Omega(s) \mathrm{P}(s)=\vec{w}(s) \wedge_{L} \vec{p}(s)
$$

for $\vec{w}(s)$ and $\vec{p} \in \mathrm{L}^{3}$, where $\vec{p}(s)$ and $\mathrm{P}$ indicate the position vector and the matrix form of the point.

i. If $\vec{w}(s)$ is a spacelike vector

$$
\mathrm{A}_{1}(s, t)=I+(\operatorname{sh} t) \Omega+(-1+\operatorname{ch} t) \Omega^{2} .
$$


ii. If $\vec{w}(s)$ is a timelike vector

$$
\mathrm{A}_{2}(s, t)=I+(\sin t) \Omega+(1-\cos t) \Omega^{2}
$$

is the orthogonal matrix defined via the semi-skew symmetric matrices $\Omega(s)$ corresponding to the vector $\vec{w}(s)=\left(w_{1}(s), w_{2}(s), w_{3}(s)\right)[10]$.

Therefore, from equations (4.3) and (4.4), we get

$$
\mathrm{A}_{1}(s, t) \vec{p}=\mathrm{A}_{1}(s, t) \mathrm{P}=\left[I+(\operatorname{sh} t) \Omega+(-1+\operatorname{ch} t) \Omega^{2}\right] \mathrm{P}
$$

and

$$
\mathrm{A}_{2}(s, t) \vec{p}=\mathrm{A}_{2}(s, t) \mathrm{P}=\left[I+(\sin t) \Omega+(1-\cos t) \Omega^{2}\right] \mathrm{P} .
$$

Also, since $\Omega \mathrm{P}=\vec{w} \wedge_{L} \vec{p}$ and $\vec{w} \wedge_{L}\left(\vec{w} \wedge_{L} \vec{p}\right)=\langle\vec{w}, \vec{p}\rangle_{L} \vec{w}-\langle\vec{w}, \vec{w}\rangle_{L} \vec{p}$ by using equations (4.3) and (4.4), we obtain

$$
\mathrm{A}_{1}(s, t) \vec{p}=\vec{p} \operatorname{ch} t+\langle\vec{w}, \vec{p}\rangle_{L} \vec{w}(1-\operatorname{ch} t)+\left(\vec{w} \wedge_{L} \vec{p}\right) \operatorname{sh} t
$$

and

$$
\mathrm{A}_{2}(s, t) \vec{p}=\vec{p} \cos t-\langle\vec{w}, \vec{p}\rangle_{L} \vec{w}(1-\cos t)+\left(\vec{w} \wedge_{L} \vec{p}\right) \sin t
$$

Definition 4.1 The two-parameter homothetic motion in a Lorenzian space along the curve $\alpha(s)$ is defined by

$$
\varphi(s, t)=h(s, t) \mathrm{A}_{1,2}(s, t) \vec{p}+\alpha(s)
$$

Let $\{\vec{T}, \vec{N}, \vec{B}\}$ be the Frenet frame of the curve $\alpha$ of the point $p$. The trajectory $\varphi(s, t)(p)$ of the point $p$ is a surface. The equation of this surface is as follows.

i. If $\vec{w}(s)$ is a spacelike vector, then from equations (4.3) and (4.7), we have

$$
\varphi_{1}(s, t)(p)=h(s, t)\left[\vec{p} \operatorname{ch} t+\langle\vec{T}, \vec{p}\rangle_{L} \vec{T}(1-\operatorname{ch} t)+\left(\vec{T} \wedge_{L} \vec{p}\right) \operatorname{sh} t\right]+\alpha(s)
$$

ii. If $\vec{w}(s)$ is a timelike vector, then from equations (4.4) and (4.8), we have

$$
\varphi_{2}(s, t)(p)=h(s, t)\left[\vec{p} \cos t-\langle\vec{T}, \vec{p}\rangle_{L} \vec{T}(1-\cos t)+\left(\vec{T} \wedge_{L} \vec{p}\right) \sin t\right]+\alpha(s)
$$

If we calculate the normals of these surfaces, there are two states depending on whether we have the timelike and spacelike cases.

1. If $\alpha(s)$ is a spacelike curve, then the tangent $\vec{T}$ is a spacelike and we have the following cases.

(a) $\vec{N}$ is a timelike and $\vec{B}$ is spacelike. Since the Frenet formulas are

$$
\vec{T}^{\prime}=k_{1} \vec{N}, \quad \vec{N}^{\prime}=k_{1} \vec{T}+k_{2} \vec{B}, \quad \vec{B}^{\prime}=k_{2} \vec{N}
$$


then from equation (4.9) we get

$$
\begin{aligned}
\varphi_{1 t}(s, t)= & h_{t}(s, t)\left[\vec{p} \operatorname{ch} t+\langle\vec{T}, \vec{p}\rangle_{L} \vec{T}(1-\operatorname{ch} t)+\left(\vec{T} \wedge_{L} \vec{p}\right) \operatorname{sh} t\right] \\
& +h(s, t)\left[\operatorname{sh} t \vec{p}+\operatorname{ch} t\left(\vec{T} \wedge_{L} \vec{p}\right)-\operatorname{sh} t\langle\vec{T}, \vec{p}\rangle_{L} \vec{T}\right]
\end{aligned}
$$

and

$$
\begin{aligned}
\varphi_{1 s}(s, t)= & h_{s}(s, t)\left[\vec{p} \operatorname{ch} t+\langle\vec{T}, \vec{p}\rangle_{L} \vec{T}(1-\operatorname{ch} t)+\left(\vec{T} \wedge_{L} \vec{p}\right) \operatorname{sh} t\right] \\
& +h(s, t)\left[\operatorname{sh} t k_{1}\left(\vec{N} \wedge_{L} \vec{p}\right)+(1-\operatorname{ch} t) k_{1}\langle\vec{N}, \vec{p}\rangle_{L} \vec{T}+(1-\operatorname{ch} t)\langle\vec{T}, \vec{p}\rangle_{L} k_{1} \vec{N}\right]+\vec{T}
\end{aligned}
$$

If we take $\vec{p}=\lambda \vec{N}$, then

$$
\begin{aligned}
& \varphi_{1 t}(s, t)=\left(h_{t} \lambda \operatorname{ch} t+h \lambda \operatorname{sh} t\right) \vec{N}+\left(h_{t} \lambda \operatorname{sh} t+h \lambda \operatorname{ch} t\right) \vec{B}, \\
& \varphi_{1 s}(s, t)=\left[-h k_{1} \lambda(1-\operatorname{ch} t)+1\right] \vec{T}+h_{s} \lambda \operatorname{ch} t \vec{N}+h_{s} \lambda \operatorname{sh} t \vec{B} .
\end{aligned}
$$

Hence, the normal of the surface drawn by the trajectory of the points $p$ is

$$
\varphi_{1 s} \wedge_{L} \varphi_{1 t}=\left|\begin{array}{ccc}
\vec{T} & -\vec{N} & \vec{B} \\
-h k_{1} \lambda(1-\operatorname{ch} t)+1 & h_{s} \lambda \operatorname{ch} t & h_{s} \lambda \operatorname{sh} t \\
0 & h_{t} \lambda \operatorname{ch} t+h \lambda \operatorname{sh} t & h_{t} \lambda \operatorname{sh} t+h \lambda \operatorname{ch} t
\end{array}\right|
$$

i.e.,

$$
\begin{aligned}
\varphi_{1 s} \wedge_{L} \varphi_{1 t}= & {\left[-h_{s} h \lambda^{2}\right] \vec{T}+\left[h k_{1} \lambda^{2}\left(h_{t} \operatorname{sh} t+h \operatorname{ch} t\right)(\operatorname{ch} t-1)+h_{t} \lambda \operatorname{sh} t+h \lambda \operatorname{ch} t\right] \vec{N} } \\
& +\left[h k_{1} \lambda^{2}\left(h_{t} \operatorname{ch} t+h \operatorname{sh} t\right)(\operatorname{ch} t-1)+h_{t} \lambda \operatorname{ch} t+h \lambda \operatorname{sh} t\right] \vec{B} .
\end{aligned}
$$

If $h(s, t)$ is a constant that is never vanishing, then the normal of this surface is in a normal plane which is perpendicular to the tangent vector field of the curve $\alpha(s)$.

Corollary 4.1 If the principal normal vector of the curve is timelike at two-parameter motion which is obtained by $\mathrm{A}_{1}(s, t)$ in $L^{3}$,

$$
\begin{aligned}
& \left\langle\varphi_{1 s} \wedge_{L} \varphi_{1 t}, \varphi_{1 s} \wedge_{L} \varphi_{1 t}\right\rangle_{L} \\
& \quad=\lambda^{2}\left[h_{s}^{2} h^{2} \lambda^{2}+h_{t}^{2}\left(1+h k_{1} \lambda(\operatorname{ch} t-1)\right)^{2}-h^{2}\left(1+h k_{1} \lambda(\operatorname{ch} t-1)\right)^{2}\right],
\end{aligned}
$$

in this expression, if $h(s, t)$ is a constant other than zero,

$$
\left\langle\varphi_{1 s} \wedge_{L} \varphi_{1 t}, \varphi_{1 s} \wedge_{L} \varphi_{1 t}\right\rangle_{L}=-h^{2} \lambda^{2}\left(1+h k_{1} \lambda(\operatorname{ch} t-1)\right)^{2}<0,
$$

and the geometric position of the selected point $p$ which is over a principal normal vector of the $\alpha$ spacelike curve is a spacelike surface.

(b) $\vec{N}$ is spacelike and $\vec{B}$ is timelike. Since the Frenet formulas are

$$
\vec{T}^{\prime}=k_{1} \vec{N}, \quad \vec{N}^{\prime}=-k_{1} \vec{T}+k_{2} \vec{B}, \quad \vec{B}^{\prime}=k_{2} \vec{N}
$$


then from equation (4.9) we get

$$
\begin{aligned}
\varphi_{1 t}(s, t)= & h_{t}(s, t)\left[\vec{p} \operatorname{ch} t+\langle\vec{T}, \vec{p}\rangle_{L} \vec{T}(1-\operatorname{ch} t)+\left(\vec{T} \wedge_{L} \vec{p}\right) \operatorname{sh} t\right] \\
& +h(s, t)\left[\operatorname{sh} t \vec{p}+\operatorname{ch} t\left(\vec{T} \wedge_{L} \vec{p}\right)-\operatorname{sh} t\langle\vec{T}, \vec{p}\rangle_{L} \vec{T}\right]
\end{aligned}
$$

and

$$
\begin{aligned}
\varphi_{1 s}(s, t)= & h_{s}(s, t)\left[\vec{p} \operatorname{ch} t+\langle\vec{T}, \vec{p}\rangle_{L} \vec{T}(1-\operatorname{ch} t)+\left(\vec{T} \wedge_{L} \vec{p}\right) \operatorname{sh} t\right] \\
& +h(s, t)\left[\operatorname{sh} t k_{1}\left(\vec{N} \wedge_{L} \vec{p}\right)+(1-\operatorname{ch} t) k_{1}\langle\vec{N}, \vec{p}\rangle_{L} \vec{T}+(1-\operatorname{ch} t)\langle\vec{T}, \vec{p}\rangle_{L} k_{1} \vec{N}\right]+\vec{T} .
\end{aligned}
$$

If we take $\vec{p}=\lambda \vec{N}$, then

$$
\begin{aligned}
& \varphi_{1 t}(s, t)=\left(h_{t} \lambda \operatorname{ch} t+h \lambda \operatorname{sh} t\right) \vec{N}-\left(h_{t} \lambda \operatorname{sh} t+h \lambda \operatorname{ch} t\right) \vec{B}, \\
& \varphi_{1 s}(s, t)=\left[h k_{1} \lambda(1-\operatorname{ch} t)+1\right] \vec{T}+h_{s} \lambda \operatorname{ch} t \vec{N}-h_{s} \lambda \operatorname{sh} t \vec{B} .
\end{aligned}
$$

Hence, the normal of the surface drawn by the trajectory of the points $p$ is

$$
\varphi_{1 s} \wedge_{L} \varphi_{1 t}=\left|\begin{array}{ccc}
\vec{T} & \vec{N} & -\vec{B} \\
h k_{1} \lambda(1-\operatorname{ch} t)+1 & h_{s} \lambda \operatorname{ch} t & -h_{s} \lambda \operatorname{sh} t \\
0 & h_{t} \lambda \operatorname{ch} t+h \lambda \operatorname{sh} t & -h_{t} \lambda \operatorname{sh} t-h \lambda \operatorname{ch} t
\end{array}\right|
$$

i.e.,

$$
\begin{aligned}
\varphi_{1 s} \wedge_{L} \varphi_{1 t}= & {\left[-h_{s} h \lambda^{2}\right] \vec{T}+\left[h k_{1} \lambda^{2}\left(h_{t} \operatorname{sh} t+h \operatorname{ch} t\right)(1-\operatorname{ch} t)+h_{t} \lambda \operatorname{sh} t+h \lambda \operatorname{ch} t\right] \vec{N} } \\
& -\left[h k_{1} \lambda^{2}\left(h_{t} \operatorname{ch} t+h \operatorname{sh} t\right)(1-\operatorname{ch} t)+h_{t} \lambda \operatorname{ch} t+h \lambda \operatorname{sh} t\right] \vec{B}
\end{aligned}
$$

Corollary 4.2 If a binormal vector of the curve is timelike a two-parameter motion is obtained by $\mathrm{A}_{1}(s, t)$ in $L^{3}$,

$$
\begin{aligned}
& \left\langle\varphi_{1 s} \wedge_{L} \varphi_{1 t}, \varphi_{1 s} \wedge_{L} \varphi_{1 t}\right\rangle_{L} \\
& \quad=\lambda^{2}\left[h_{s}^{2} h^{2} \lambda^{2}-h_{t}^{2}\left(1+h k_{1} \lambda(1-\operatorname{ch} t)\right)^{2}+h^{2}\left(1+h k_{1} \lambda(1-\operatorname{ch} t)\right)^{2}\right],
\end{aligned}
$$

in this expression, if $h(s, t)$ is a constant other than zero,

$$
\left\langle\varphi_{1 s} \wedge_{L} \varphi_{1 t}, \varphi_{1 s} \wedge_{L} \varphi_{1 t}\right\rangle_{L}=h^{2} \lambda^{2}\left(1+h k_{1} \lambda(\operatorname{ch} t-1)\right)^{2}>0,
$$

the geometric position of the selected point $p$ which is over a principal binormal vector of the $\alpha$ spacelike curve is a timelike surface.

2. If $\alpha(s)$ is a timelike curve, then the tangent $\vec{T}$ is timelike and we have the following case.

$\vec{N}$ and $\vec{B}$ are both spacelike. Since the Frenet formulas are

$$
\vec{T}^{\prime}=k_{1} \vec{N}, \quad \vec{N}^{\prime}=k_{1} \vec{T}+k_{2} \vec{B}, \quad \vec{B}^{\prime}=-k_{2} \vec{N}
$$


then from equation (4.10) we get

$$
\begin{aligned}
\varphi_{2 t}(s, t)= & h_{t}(s, t)\left[\vec{p} \cos t+\langle\vec{T}, \vec{p}\rangle_{L} \vec{T}(1-\cos t)+\left(\vec{T} \wedge_{L} \vec{p}\right) \sin t\right] \\
& +h(s, t)\left[-\vec{p} \sin t-\langle\vec{T}, \vec{p}\rangle_{L} \vec{T} \sin t+\left(\vec{T} \wedge_{L} \vec{p}\right) \cos t\right]
\end{aligned}
$$

and

$$
\begin{aligned}
\varphi_{2 s}(s, t)= & h_{s}(s, t)\left[\vec{p} \cos t+\langle\vec{T}, \vec{p}\rangle_{L} \vec{T}(1-\cos t)+\left(\vec{T} \wedge_{L} \vec{p}\right) \sin t\right] \\
& +h(s, t)\left[k_{1} \sin t\left(\vec{N} \wedge_{L} \vec{p}\right)-(1-\cos t) k_{1}\langle\vec{N}, \vec{p}\rangle_{L} \vec{T}-(1-\cos t)\langle\vec{T}, \vec{p}\rangle_{L} k_{1} \vec{N}\right]+\vec{T} .
\end{aligned}
$$

If we take $\vec{p}=\lambda \vec{N}$, then

$$
\begin{aligned}
& \varphi_{2 t}(s, t)=\left(h_{t} \lambda \cos t-h \lambda \sin t\right) \vec{N}+\left(h_{t} \lambda \sin t+h \lambda \cos t\right) \vec{B}, \\
& \varphi_{2 s}(s, t)=\left[1-h k_{1} \lambda(1-\cos t)\right] \vec{T}+h_{s} \lambda \cos t \vec{N}+h_{s} \lambda \sin t \vec{B} .
\end{aligned}
$$

Hence, the normal of the surface drawn by the trajectory of the points $p$ is

$$
\varphi_{2 s} \wedge_{L} \varphi_{2 t}=\left|\begin{array}{ccc}
-\vec{T} & \vec{N} & \vec{B} \\
1+h k_{1} \lambda(\cos t-1) & h_{s} \lambda \cos t & h_{s} \lambda \sin t \\
0 & h_{t} \lambda \cos t-h \lambda \sin t & h_{t} \lambda \sin t+h \lambda \cos t
\end{array}\right|
$$

i.e.,

$$
\begin{aligned}
\varphi_{2 s} \wedge_{L} \varphi_{2 t}= & {\left[h_{s} h \lambda^{2}\right] \vec{T}-\left[h k_{1} \lambda^{2}\left(h_{t} \sin t+h \cos t\right)(\cos t-1)+h_{t} \lambda \sin t+h \lambda \cos t\right] \vec{N} } \\
& +\left[h k_{1} \lambda^{2}\left(h_{t} \cos t-h \sin t\right)(\cos t-1)+h_{t} \lambda \cos t-h \lambda \sin t\right] \vec{B}
\end{aligned}
$$

Corollary 4.3 A two-parameter motion is obtained by $\mathrm{A}_{2}(s, t)$ in $L^{3}$,

$$
\begin{aligned}
& \left\langle\varphi_{2 s} \wedge_{L} \varphi_{2 t}, \varphi_{2 s} \wedge_{L} \varphi_{2 t}\right\rangle_{L} \\
& \quad=\lambda^{2}\left[-h_{s}^{2} h^{2} \lambda^{2}+h_{t}^{2}\left(1+h k_{1} \lambda(\cos t-1)\right)^{2}+h^{2}\left(1+h k_{1} \lambda(\cos t-1)\right)^{2}\right],
\end{aligned}
$$

in this expression, if $h(s, t)$ is a constant other than zero,

$$
\left\langle\varphi_{2 s} \wedge_{L} \varphi_{2 t}, \varphi_{2 s} \wedge_{L} \varphi_{2 t}\right\rangle_{L}=\lambda^{2} h^{2}\left(1+h k_{1} \lambda(\cos t-1)\right)^{2}>0,
$$

the geometric position of the selected point $p$ which is over a principal binormal vector of the $\alpha$ timelike curve is a timelike surface.

\section{Parametrizations of trajectory surfaces}

In this section, we find some parametrizations of the trajectory surfaces obtained from two-parameter motions in a Lorentzian space. 


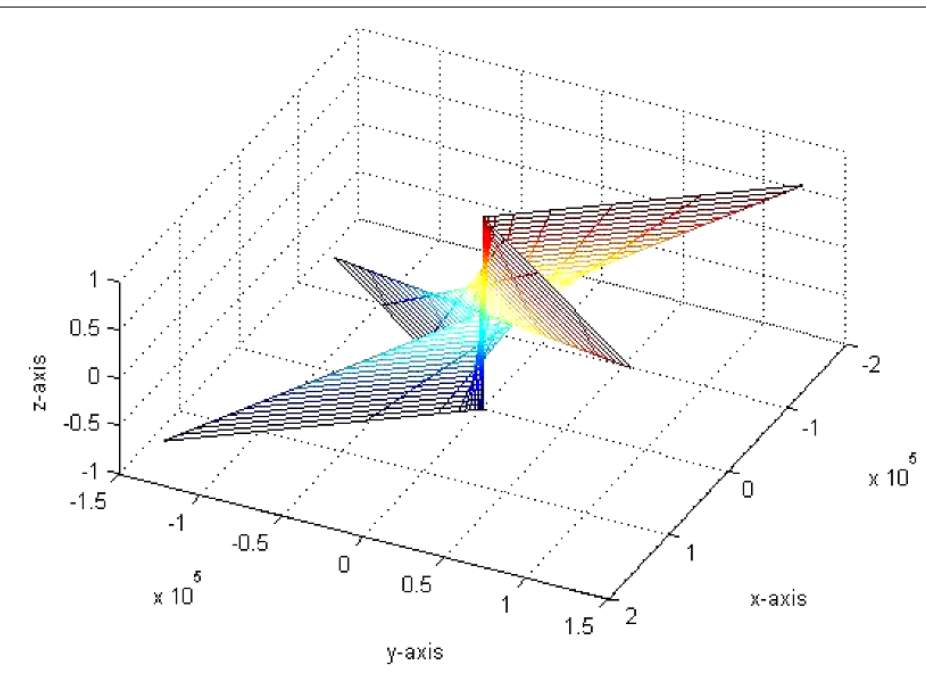

Figure 1 Spacelike homothetic cylinder.

\subsection{Cylinder surface}

\subsection{1 $\alpha(s)$ is spacelike}

Assume that $\alpha(s)=(0,0, s)$ and $p=\left(p_{1}, p_{2}, p_{3}\right) \in L^{3}$. Substituting these into equation (4.9), we get

$$
\varphi(s, t)(p)=\left(h p_{1} \operatorname{ch} t+h p_{2} \operatorname{sh} t, h p_{2} \operatorname{ch} t+h p_{1} \operatorname{sh} t, h p_{3}+s\right) .
$$

As a special case, if $p=\left(p_{1}, p_{2}, 0\right)$, we obtain

$$
\varphi(s, t)(p)=\left(h p_{1} \operatorname{ch} t+h p_{2} \operatorname{sh} t, h p_{2} \operatorname{ch} t+h p_{1} \operatorname{sh} t, s\right) .
$$

For $p_{1}=r \operatorname{sh} \theta$ and $p_{2}=r \operatorname{ch} \theta$, we get

$$
\varphi(s, t)(p)=(h r \operatorname{sh} \theta \operatorname{ch} t+h r \operatorname{ch} \theta \operatorname{sh} t, h r \operatorname{ch} \theta \operatorname{ch} t+h r \operatorname{sh} \theta \operatorname{sh} t, s),
$$

that is

$$
\varphi(s, t)(p)=(h r \operatorname{sh}(\theta+t), h r \operatorname{ch}(\theta+t), s) .
$$

Example 5.1 Let $-1<s<1,-2 \pi<t, \theta<2 \pi$ and $h(s, t)=s+\sin t \cos t$ in equation (5.1), then we can obtain the homothetic cylinder surface given in Figure 1.

Example 5.2 If we take $h(s, t)=1$ in equation (5.1) the spacelike cylinder surface is obtained as given in Figure 2.

\section{$5.1 .2 \alpha(s)$ is timelike}

Assume that $\alpha(s)=(s, 0,0)$ and $p=\left(p_{1}, p_{2}, p_{3}\right) \in L^{3}$. Substituting these into the equation (4.10), we get

$$
\varphi_{2}(s, t)(p)=\left(-h p_{1}+s, h p_{2} \cos t-h p_{3} \sin t, h p_{3} \cos t+h p_{2} \sin t\right) .
$$




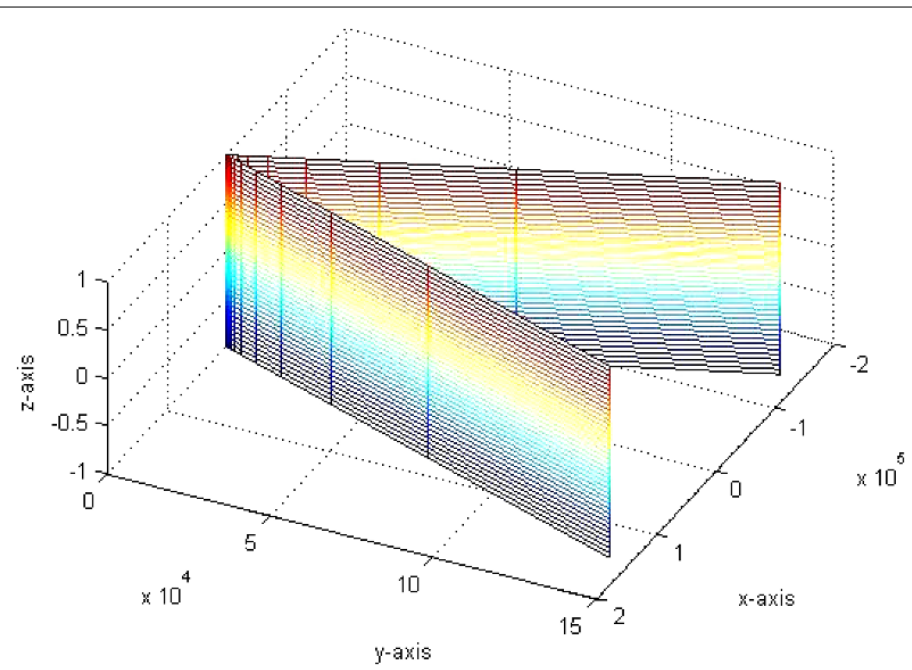

Figure 2 Spacelike cylinder.

As a special case, if $p=\left(0, p_{2}, p_{3}\right)$, we obtain

$$
\varphi(s, t)(p)=\left(s, h p_{2} \cos t-h p_{3} \sin t, h p_{3} \cos t+h p_{2} \sin t\right) .
$$

For $p_{2}=r \cos \theta$ and $p_{3}=r \sin \theta$, we get

$$
\varphi(s, t)(p)=(s, h r \cos \theta \cos t-h r \sin \theta \sin t, h r \sin \theta \cos t+h r \cos \theta \sin t),
$$

that is

$$
\varphi(s, t)(p)=(s, h r \cos (\theta+t), h r \sin (\theta+t)) .
$$

Example 5.3 Let $-1<s<1,0<t, \theta<\pi$ and $h(s, t)=s+\sin t \cos t$ in the equation (5.2), then we can obtain the homothetic cylinder surface given in Figure 3.

Example 5.4 If we take $h(s, t)=1$ in equation (5.2) the timelike cylinder surface is obtained as given in Figure 4.

\subsection{Hyperboloid surface}

\section{$5.2 .1 \alpha(s)$ is spacelike}

Let $\alpha(s)=(0,0, s)$ and $p(s)=(1, s, 0)$; substituting these into equation (4.9), we get

$$
\varphi(s, t)(p)=(h \operatorname{ch} t-h s \operatorname{sh} t, h s \operatorname{ch} t+h \operatorname{sh} t, s) .
$$

Example 5.5 In equation (5.3) if $-4<s<4,-\pi<t<\pi$ and $h(s, t)=s+\sin t \cos t$ are given, then a spacelike homothetic hyperboloid surface is obtained as given in Figure 5.

Example 5.6 In equation (5.3) if $h(s, t)=1$ is taken, then a spacelike hyperboloid surface is obtained as given by Figure 6 . 


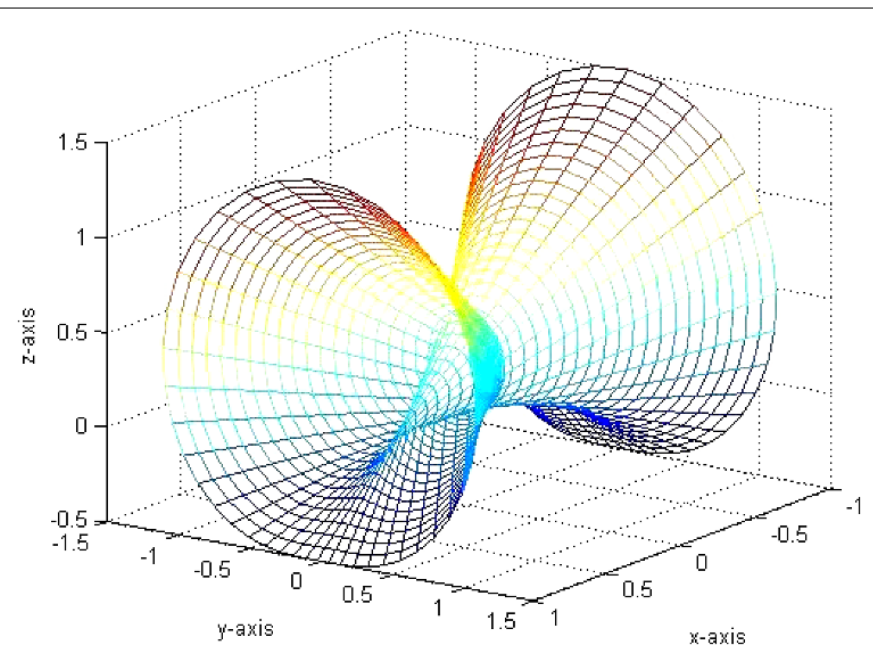

Figure 3 Timelike homothetic cylinder.

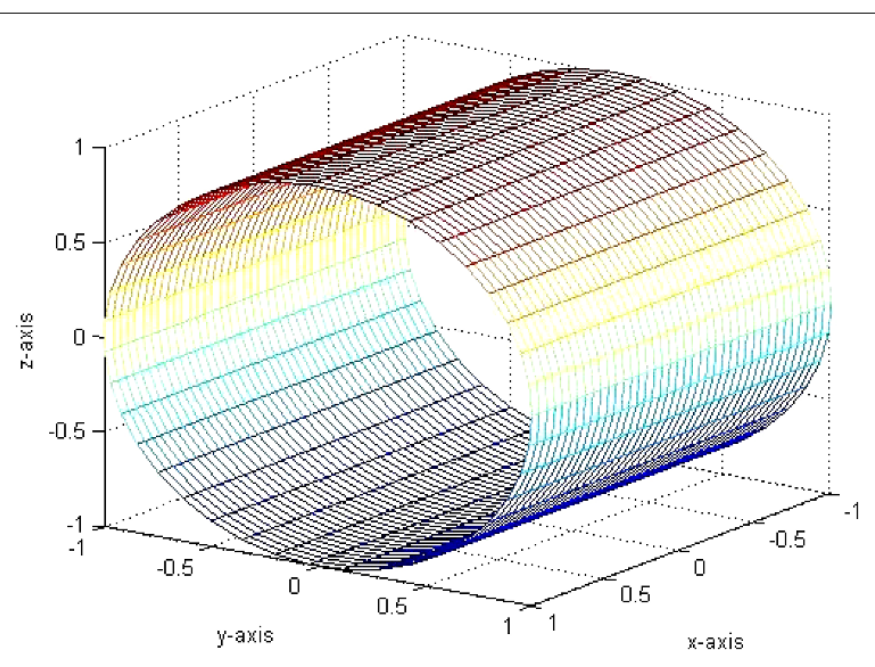

Figure 4 Timelike cylinder.

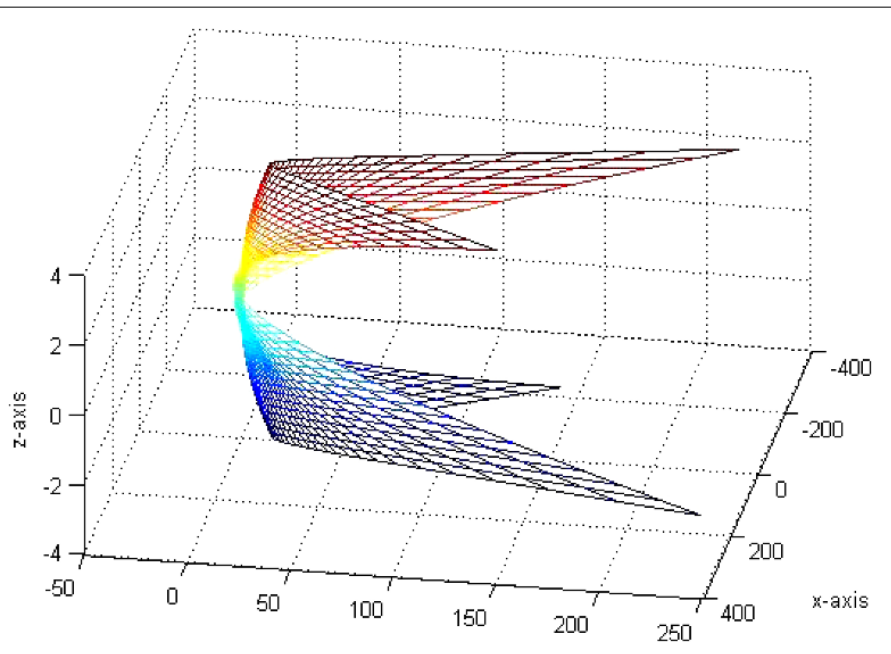

Figure 5 Spacelike homothetic hyperboloid. 


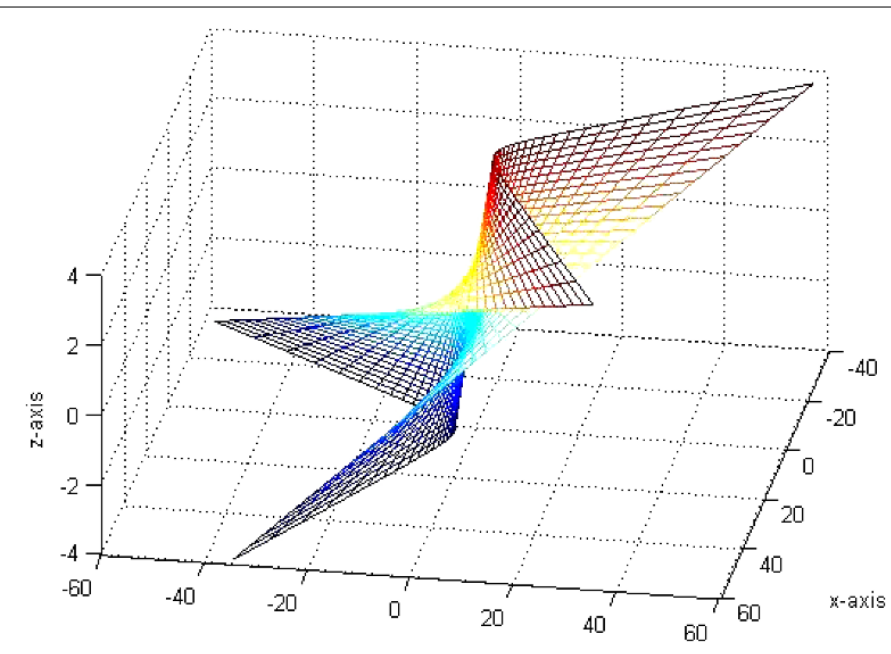

Figure 6 Spacelike hyperboloid.

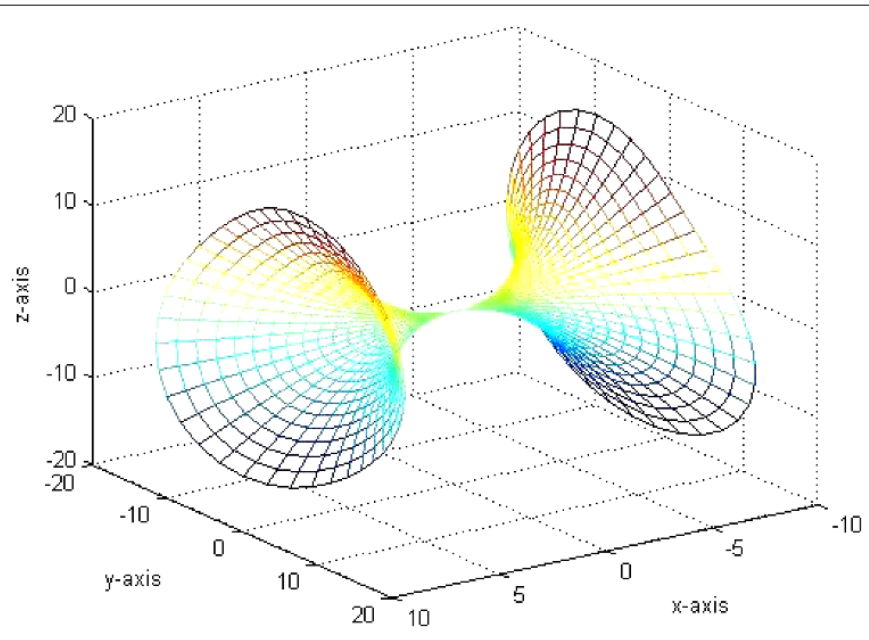

Figure 7 Timelike homothetic hyperboloid.

\subsection{2 $\alpha(s)$ is timelike}

Let $\alpha(s)=(0,0, s)$ and $p(s)=(1, s, 0)$; substituting these into equation (4.10), we get

$$
\varphi_{2}(s, t)(p)=(h+s, h s \cos t, h s \sin t) .
$$

Example 5.7 In equation (5.4) if $-4<s<4,-\pi<t<\pi$ and $h(s, t)=s+\sin t \cos t$ are given, then a timelike homothetic hyperboloid surface is obtained as given in Figure 7.

Example 5.8 In equation (5.4) if $h(s, t)=1$ is taken, then a timelike hyperboloid surface is obtained as given in Figure 8 .

\subsection{Tor surface}

\subsection{1 $\alpha(s)$ is spacelike}

Let $\vec{T}$ be spacelike, $\vec{N}$ timelike and $\vec{B}$ spacelike. Let the curve $\alpha(s)=(r \sin \theta, r \cos \theta, 0)$ be a Lorentz circle with radius $r$ on the $x y$-plane. Then the Frenet frame of the curve $\alpha(s)$ at 


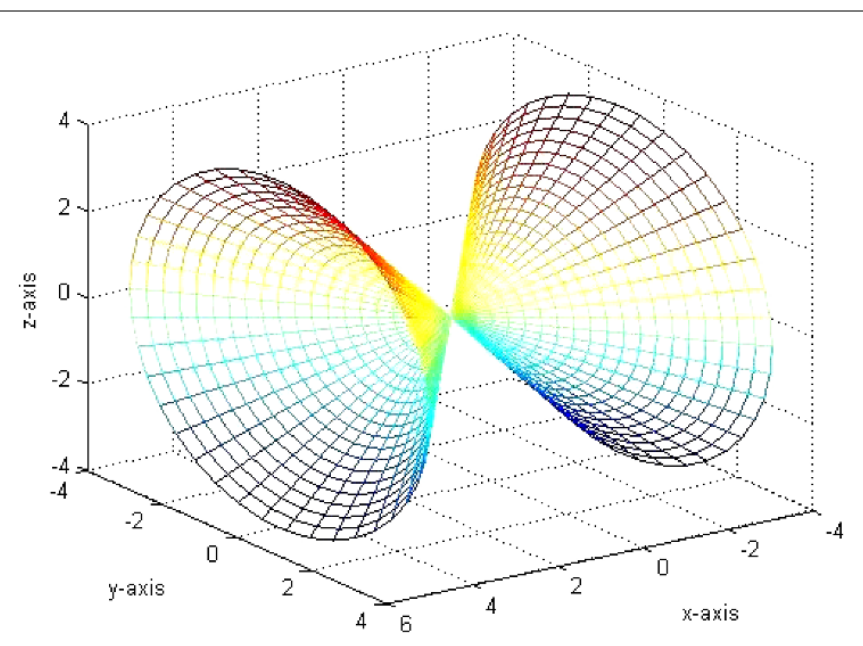

Figure 8 Timelike hyperboloid.

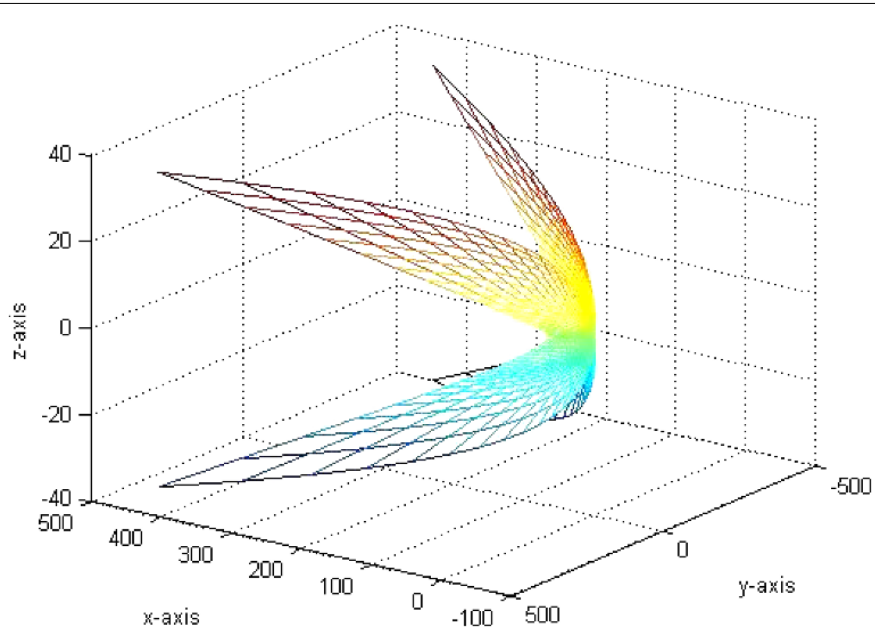

Figure 9 Spacelike homothetic tor surface.

the point $p$ is

$$
\vec{T}=(\operatorname{sh} s, \operatorname{ch} s, 0), \quad \vec{N}=(\operatorname{ch} s, \operatorname{sh} s, 0), \quad \vec{B}=(0,0,-1)
$$

and for $\vec{p}=\lambda \vec{N}$, substituting these equations into equation (4.9), we obtain an equation of the tor surface as follows:

$$
\varphi(s, t)(p)=(\operatorname{ch} s[r+h \lambda \operatorname{ch} t], \operatorname{sh} s[r+h \lambda \operatorname{ch} t],-h \lambda \operatorname{sh} t) .
$$

Example 5.9 In equation (5.5) if $-\pi<s, t<\pi$ and $h(s, t)=s+\sin t \cos t$ are given, then a spacelike homothetic tor surface is obtained as drawn in Figure 9.

Example 5.10 In equation (5.5) if $h(s, t)=1$ is taken, then a spacelike tor surface is obtained as drawn in Figure 10. 


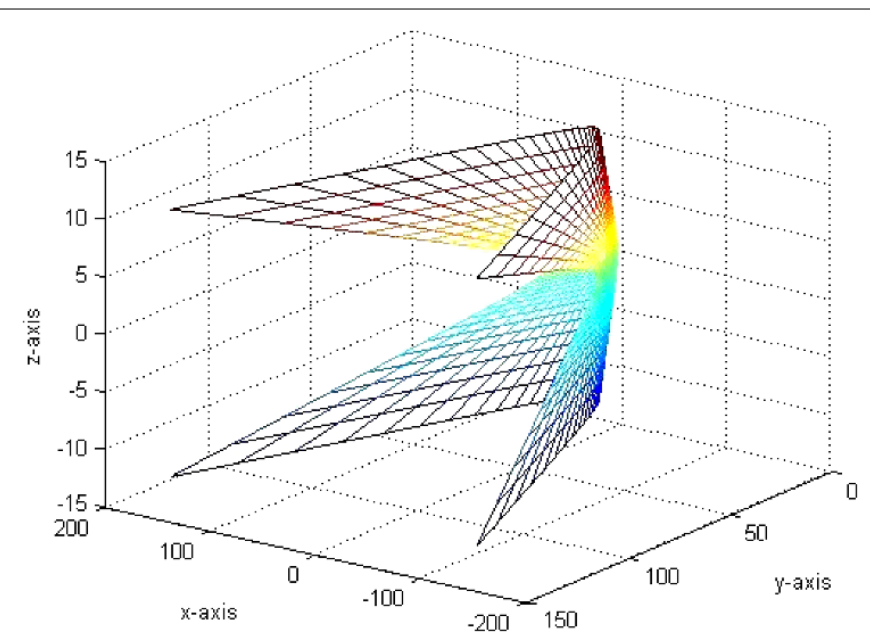

Figure 10 Spacelike tor surface.

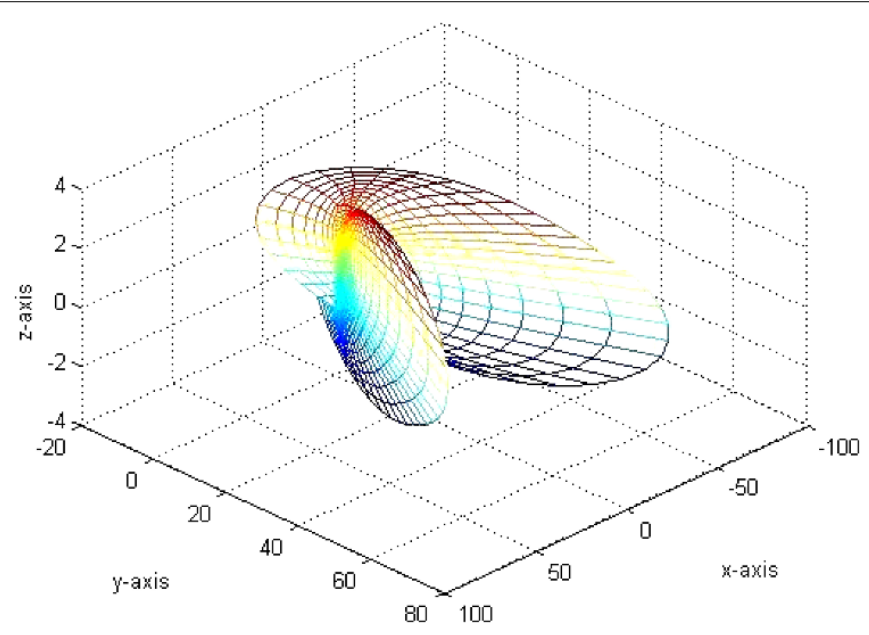

Figure 11 Timelike homothetic tor surface.

5.3.2 $\alpha(s)$ is timelike

Let the curve $\alpha(s)=(r \sin \theta, r \cos \theta, 0)$ be a Lorentz circle with radius $r$ on the $x y$-plane. Then, the Frenet frame of the curve $\alpha(s)$ at the point $p$ is

$$
\vec{T}=(\operatorname{ch} s, \operatorname{sh} s, 0), \quad \vec{N}=(\operatorname{sh} s, \operatorname{ch} s, 0), \quad \vec{B}=(0,0,1)
$$

and for $\vec{p}=\lambda \vec{N}$, substituting these equations into equation (4.10), we obtain an equation of the tor surface as follows:

$$
\varphi_{2}(s, t)(p)=(\operatorname{sh} s[r+h \lambda \cos t], \operatorname{ch} s[r+h \lambda \cos t], h \lambda \sin t) .
$$

Example 5.11 In equation (5.6) if $-\pi<s, t<\pi$ and $h(s, t)=s+\sin t \cos t$ are given, then a timelike homothetic tor surface is obtained as drawn in Figure 11. 


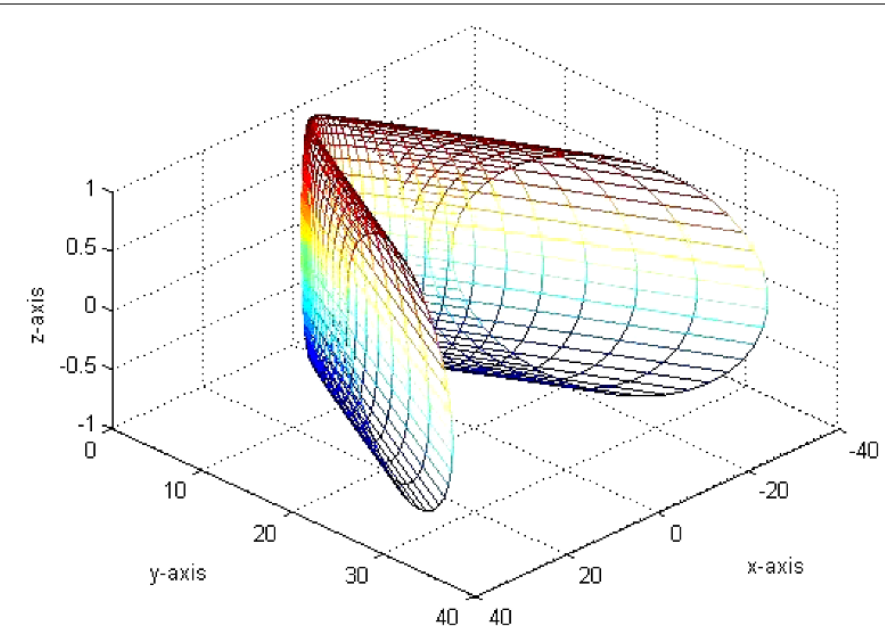

Figure 12 Timelike tor surface.

Example 5.12 In equation (5.6) if $h(s, t)=1$ is taken, then a timelike tor surface is obtained as drawn in Figure 12.

\section{Conclusion}

The results we have presented deal with Lorentzian homothetic motions in which the position of the moving object depends on two parameters. The hodographs of two-parameter Lorentzian homothetic motions were obtained. A hodograph is the locus of the end points of the velocity of a particle and it is the solution of the first order equation which is Newton's Law. The locus of the hodograph of a Lorentzian homothetic motion was found as a hyperbola in this study.

Also this paper deals with trajectory surfaces (cylinder, hyperboloid and tor surfaces) generated by a point, the moving body, and figures of these surfaces were drawn by using MATLAB software.

\section{Competing interests}

The authors declare that they have no competing interests.

Authors' contributions

Each of the authors read and approved the final version of the manuscript.

\section{Acknowledgements}

We would like to thank to the reviewers whose careful reading, helpful suggestions and valuable comments helped us to improve the manuscript.

Received: 11 October 2013 Accepted: 6 January 2014 Published: 27 Jan 2014

\section{References}

1. Farouki, RT: The approximation of non-degenerate offset surface. Comput. Aided Geom. Des. 3, 15-43 (1986)

2. Papaioannou, SG, Kiritsis, D: An application of Bertrand curves and surfaces to CAD/CAM. Comput. Aided Des. 17(8), 348-352 (1985)

3. Chen, YJ, Ravani, B: Offsets surface generation and contouring in computer aided design. J. Mech. Transm. Autom. Des. 109, 133-142 (1987)

4. Müller, HR: Kinematik. Sammlung Göschen, vol. 584/584a. de Gruyter, Berlin (1963)

5. Bottema, O, Roth, B: Theoretical Kinematics. North Holland, Amsterdam (1979)

6. Karger, A, Novák, J: Space Kinematics and Lie Groups. Breach Science Publishers, New York (1985)

7. Ergin, AA: The kinematic geometry on the Lorentz plane. PhD Thesis, Ankara University (1989)

8. Karacan, MK, Yaylı, Y: Special two parameter motion in Lorentzian plane. Thai J. Math. 2(2), 239-246 (2004) 
9. O'Neill, B: Semi-Riemannian Geometry with Applications to Relativity. Academic Press, London (1963)

10. Kula, L, Yayli, Y: Dual split quaternions and screw motions in Minkowski 3-space. Iran. J. Sci. Technol., Trans. A, Sci. 30 245-258 (2006)

10.1186/1687-1847-2014-42

Cite this article as: Çelik et al.: On the two-parameter Lorentzian homothetic motions. Advances in Difference Equations 2014, 2014:42

Submit your manuscript to a SpringerOpen ${ }^{\circ}$ journal and benefit from:

- Convenient online submission

- Rigorous peer review

- Immediate publication on acceptance

Open access: articles freely available online

- High visibility within the field

- Retaining the copyright to your article

Submit your next manuscript at $>$ springeropen.com 